\title{
A decision analysis of surveillance for colorectal cancer in ulcerative colitis
}

\author{
F Delcò, A Sonnenberg
}

\begin{abstract}
Background-Patients with long standing, extensive ulcerative colitis have an increased risk of developing colorectal cancer.

Aims-To assess the feasibility of surveillance colonoscopy in preventing death from colorectal cancer.

Patients-A hypothetical cohort of patients with chronic ulcerative colitis.

Methods-The benefits of life years saved were weighted against the costs of biannual colonoscopy and proctocolectomy, and the terminal care of patients dying from colorectal cancer. Two separate Markov processes were modelled to compare the cost-benefit relation in patients with or without surveillance. The cumulative probability of developing colorectal cancer served as a threshold to determine which of the two management strategies is associated with a larger net benefit.

Results-If the cumulative probability of colorectal cancer exceeds a threshold value of $27 \%$, surveillance becomes more beneficial than no surveillance. The threshold is only slightly smaller than the actual cumulative cancer rate of $30 \%$. Variations of the assumptions built into the model can raise the threshold above or lower it far below the actual rate. If several of the assumptions are varied jointly, even small changes can lead to extreme threshold values.

Conclusions-It is not possible to prove that frequent colonoscopies scheduled at regular intervals are an effective means to manage the increased risk of colorectal cancer associated with ulcerative colitis. (Gut 2000;46:500-506)
\end{abstract}

Keywords: cancer screening; colorectal cancer; health economics; medical decision analysis; surveillance colonoscopy; ulcerative colitis

Department of Veterans Affairs Medical Center and

The University of New Mexico, Albuquerque, New Mexico, USA

$\mathrm{F}$ Delcò

A Sonnenberg

Correspondence to: Dr A Sonnenberg, Department of Veterans Affairs Medical Center $111 \mathrm{~F}$, 1501 San Pedro Drive SE, Albuquerque, New Mexico 87108, USA

Accepted for publication 22 October 1999 three to five years can lead to full blown cancer. The presence of dysplasia in biopsy specimens obtained through surveillance colonoscopy suggests that a patient undergoes prophylactic proctocolectomy to achieve primary cancer prevention. Little evidence exists, however, that surveillance is truly efficacious and cost effective in preventing deaths from colorectal cancer. No randomised controlled trial has been performed in the attempt to define the role of surveillance colonoscopy in colorectal cancer prevention. The prevalence of extensive ulcerative colitis is relatively small, and even large medical centres committed to the study of inflammatory bowel disease rarely follow more than 200 patients at any given point in time. It is not likely that a randomised clinical trial will be conducted soon to provide conclusive evidence in favour of or against surveillance. Considering the obstacles to a clinical resolution, the aim of the present study was to utilise the techniques of medical decision analysis in trying to assess the feasibility and usefulness of surveillance.

\section{Methods}

In the surveillance of patients with ulcerative colitis, the benefit of life years saved needs to be weighted against the costs of colonoscopy, proctocolectomy, and the terminal care of patients dying from colorectal cancer. Two separate Markov processes were modelled to compare the cost-benefit relation in patients with or without surveillance by biannual colonoscopy. The cumulative probability of developing colorectal cancer served as a threshold to determine which of the two management strategies is associated with a larger net benefit. ${ }^{12}$

MARKOV MODEL

The present analysis deals with a hypothetical cohort of patients, who are presently 50 years old and who were first diagnosed with ulcerative colitis 10 years ago. Medical events are modelled as transitions of patients among a predefined set of health states, the occurrence of each transition being governed by a probability value (fig 1). ${ }^{13}$ The time frame of the analysis is divided into equal increments of two years duration, during which patients may cycle from one state to another. The white rectangles of fig 1 represent permanent states in the true sense of a Markov process, as subjects remain in these states at least for the complete

Abbreviations used in this paper: CRC, colorectal cancer; DYS, dysplasia; HRQL, health related quality of life. 


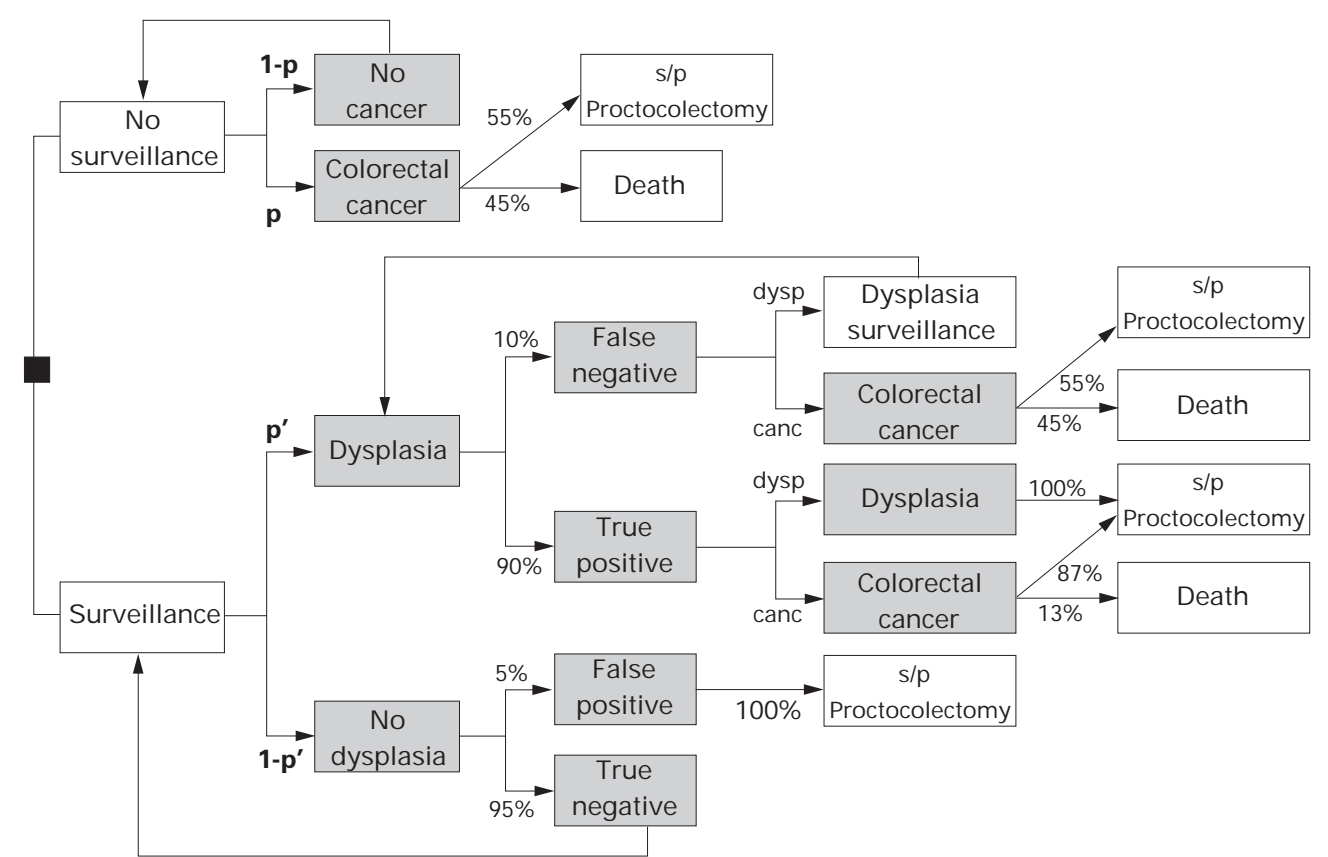

Figure 1 Markov state diagram of surveillance and no surveillance for colorectal cancer. The white rectangles represent true Markov states where subjects remain for the complete length of a cycle. The shaded rectangles represent intermediate states, which subjects can enter and leave during one cycle before settling in a true Markov state. The transition p represents the incidence rate of cancer per cycle, $p^{\prime}$ represent the incidence of high grade dysplasia. In patients with high grade dysplasia, dysp represents the fraction of patients with dysplasia alone, while canc represents the fraction of high grade dysplasia accompanied by cancer.

length of a two year cycle. The grey rectangles symbolise intermediate states of patients with or without surveillance. Subjects can enter and leave these intermediate states during one cycle before settling in a permanent Markov state.

The upper of part of fig 1 shows the natural history in patients without surveillance. Patients may develop cancer with a probability $\mathrm{p}$ or may stay cancer free with a probability $1-\mathrm{p}$. In the case of colorectal cancer, patients may survive after a proctocolectomy or die from colorectal cancer. After a cycle of two years, patients without cancer are re-entered into the analysis and are reassessed for the potential development of colorectal cancer. The same pathway is followed during each subsequent cycle.

The lower part of fig 1 shows the natural history in patients undergoing surveillance. The probability p' of developing high grade dysplasia is higher than the probability $\mathrm{p}$ of developing cancer. In patients with high grade dysplasia, the outcome of surveillance is determined by the rate of false negative and true positive

Table 1 Baseline assumptions and range tested in the sensitivity analysis

\begin{tabular}{lll}
\hline & Baseline model & Sensitivity analysis \\
\hline $\begin{array}{l}\text { Sensitivity in detecting dysplasia and CRC } \\
\text { Specificity of surveillance in detecting dysplasia }\end{array}$ & $90 \%$ & $50-100 \%$ \\
$\quad$ and CRC & $95 \%$ & $80-100 \%$ \\
Case fatality rate of CRC in surveillance & $13 \%$ & $5-45 \%$ \\
Health related quality of life after proctocolectomy & $95 \%$ & $85-100 \%$ \\
Surveillance interval & $2 \mathrm{y}$ & $1-5 \mathrm{y}$ \\
Time lag between dysplasia and CRC & $3 \mathrm{y}$ & $1-5 \mathrm{y}$ \\
Costs per surveillance colonoscopy & $\$ 911$ & $\$ 200-\$ 1500$ \\
Costs of proctocolectomy & $\$ 8300$ & $\$ 4150-\$ 16600$ \\
Costs of terminal care per patient with CRC & $\$ 34100$ & $\$ 17050-\$ 68200$ \\
Average annual income & $\$ 24294$ & $\$ 10000-\$ 50000$ \\
Annual discount rate & $3 \%$ & - \\
\hline
\end{tabular}

CRC, colorectal cancer. test results. If a false negative test concerns the presence of dysplasia alone, patients will continue to be followed in the surveillance programme and will be given another chance of a correct diagnosis during the next cycle of surveillance. A false negative test of high grade dysplasia accompanied by cancer will result in survival through timely proctocolectomy or death from colorectal cancer. The outcome of proctocolectomy in a patient with a true positive test also depends on whether the patient has dysplasia alone or dysplasia accompanied by cancer. Lastly, in patients without dysplasia, colonoscopy plus biopsy can result in false positive and true negative tests. A false positive test leads to proctocolectomy, while patients with a true negative test continue to be followed by surveillance colonoscopy. In addition to the states shown in fig 1 , the population in each state is subjected to natural attrition by the annual age specific death rate of the US population. ${ }^{14}$

TRANSITION PROBABILITIES

Table 1 lists the transition probabilities built into the model and their range tested in the sensitivity analyses. As no data are available for the sensitivity and specificity of repetitive colonoscopy plus biopsy in detecting cancer and dysplasia, we rely on previously used expert opinions and on the reported coincidence of cancer and dysplasia. ${ }^{9}{ }^{15} 16$ These rates are inflated to estimate rather sensitive and specific test characteristics of surveillance colonoscopy plus multiple biopsies. The cancer related death rates of $13 \%$ and $45 \%$ in patients with or without surveillance, respectively, are taken from a report of Connell et al. ${ }^{17}$ The mortality associated with proctocolectomy is 


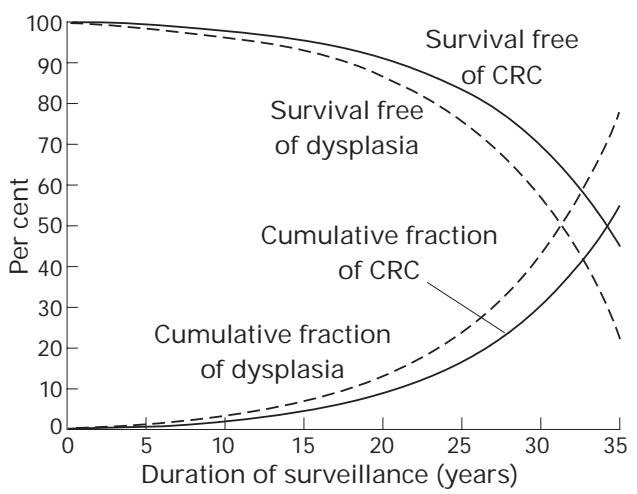

Figure 2 Cumulative fraction of patients with dysplasia or colorectal cancer (CRC), and surviving fraction of patients remaining free of dysplasia or colorectal cancer. Cumulative fractions were calculated using equations (1) and (3) with $\% C R C_{30,}=30 \%$, lag $=$ three years, survival $=100 \%-$ cumulative fraction.

assumed to be negligible in comparison with the cancer related death rates.

Between the second and fourth decade after the first diagnosis of ulcerative colitis, the cumulative fraction of patients with colorectal cancer rises in an exponential fashion $(\% \mathrm{CRC}): \% \mathrm{CRC}=\mathrm{e}^{\mathrm{k} . \mathrm{time}}-100 \%(1)$

If the cumulative fraction \%CRC after a given time is known, equation (1) can be solved to calculate the time constant $\mathrm{k}$. For instance, data from the literature suggest that $30 \%$ of all patients with pancolitis will have developed cancer over a time period of 30 years between the beginning of the second and the end of the fourth decade after the onset of the disease. ${ }^{4-7}$ The time dependent increase in the occurrence of colorectal cancer corresponds to the transition probability $\mathrm{p}$ for the development of cancer per one cycle length. It is calculated as the difference between two values of equation (1) at two consecutive time points one cycle length apart: $p=\% \mathrm{CRC}_{2}-\% \mathrm{CRC}_{1}=\mathrm{e}^{\mathrm{k} \text {.time }}$ $\left(\mathrm{e}^{\mathrm{k} . \text { cycle }}-100 \%\right)(2)$

From long term follow up data, Lashner et al have estimated an average time lag of three years between the first occurrence of dysplasia and the subsequent development of cancer. ${ }^{18}{ }^{19}$ Hence, the time dependence of the cumulative probability of dysplasia can be modelled as: $\%$ DYS $=\mathrm{e}^{\mathrm{k}(\text { time+lag })}-100 \%(3)$

where the time constant $\mathrm{k}$ is given by equation (1). The time dependent increase in the occurrence of dysplasia corresponds to: $\mathrm{p}^{\prime}=\% \mathrm{DYS}_{2}$ $-\%$ DYS $_{1}=\mathrm{e}^{\mathrm{k}(\text { time+lag) }}\left(\mathrm{e}^{\mathrm{k} . \text { cycle }}-100 \%\right)(4)$

Figure 2 shows the time dependent increase in the occurrence of dysplasia or cancer and the survival function of patients who remain free of either one. The proportional breakdown of false negative and true positive colonoscopies into dysplasia (dysp) and cancer (canc), as depicted in the Markov process of fig 1, is calculated as the proportional difference between $\mathrm{p}^{\prime}$ and $\mathrm{p}$ from equations (4) and (2), respectively: canc $=\left(\mathrm{p}^{\prime}-\mathrm{p}\right) / \mathrm{p}^{\prime}$ and dysp $=1-$ canc $=$ $\mathrm{p} / \mathrm{p}^{\prime}(5)$

The outcomes of the two competing management strategies of surveillance versus no surveillance depend on the fraction $p$ of patients with cancer and the fraction $\mathrm{p}^{\prime}$ of patients with dysplasia. As shown by equations
(1) to (4), both fractions depend on the time constant $\mathrm{k}$ calculated from the cumulative fraction of patients with colorectal cancer. Therefore, a threshold value of cumulative cancer rate can be found through iteration, for which both management strategies yield an identical net benefit. The threshold value will change in relation to the transition probabilities or the assumptions regarding costs and benefits built into the model. A very low threshold would speak much in favour of surveillance, as this value would be easily surpassed by the actual cumulative lifetime probability of developing colorectal cancer. The higher the threshold value, the less convincing the argument would be in favour of endoscopic surveillance.

\section{COSTS AND DISCOUNTING}

Table 1 summarises the cost estimates used in the present analysis. The expenses for surveillance cover both physician fees and facility costs arising from colonoscopy plus histological examination. The expenses also include potential complications of colonoscopy requiring hospitalisation, such as bleeding and perforation. The two complications are assumed to occur in $0.15 \%$ and $0.2 \%$ of the procedures, respectively. ${ }^{20}$ The costs represent the payments by the US Health Care Finance Administration (HCFA) during the fiscal year 1998. The cost estimates of proctocolectomy and terminal care of patients with colorectal cancer are taken from a publication by Provenzale et $a .^{21}$

The benefit of surveillance is measured in terms of life years gained. The status post proctocolectomy is weighted by a percentage decrease in health related quality of life (HRQL). Its value depends, in part, on whether HRQL is assessed by previously asymptomatic patients or patients with severe symptoms of pancolitis or colorectal cancer. The HRQL associated with prophylactic proctocolectomy has not been analysed. After proctocolectomy for persisting severe symptoms of colitis it was found to vary between $80 \%$ and $100 \% .{ }^{22}{ }^{23}$ For the purpose of the present analysis, a baseline estimate of $95 \%$ is assumed. The value of life is estimated by the human capital approach, using the average annual income in the United States. ${ }^{24}{ }^{25}$ Future costs and benefits are both discounted by an annual rate of $3 \%{ }^{26}$

\section{Results}

Under baseline assumptions, the two strategies of surveillance or no surveillance yield an identical net benefit, if the cumulative rate of colorectal cancer reaches a value of $\%$ CRC $=27 \%$ after 30 years of surveillance. If the actual cumulative rate of colorectal cancer exceeds this value, management of ulcerative colitis by biannual surveillance colonoscopy becomes more beneficial than no surveillance. Vice versa, an expected cumulative rate lower than this threshold would speak against surveillance. As indicated above, the cumulative probability of colorectal cancer reported in the literature exceeds this baseline threshold value and would thus seem to support a management 

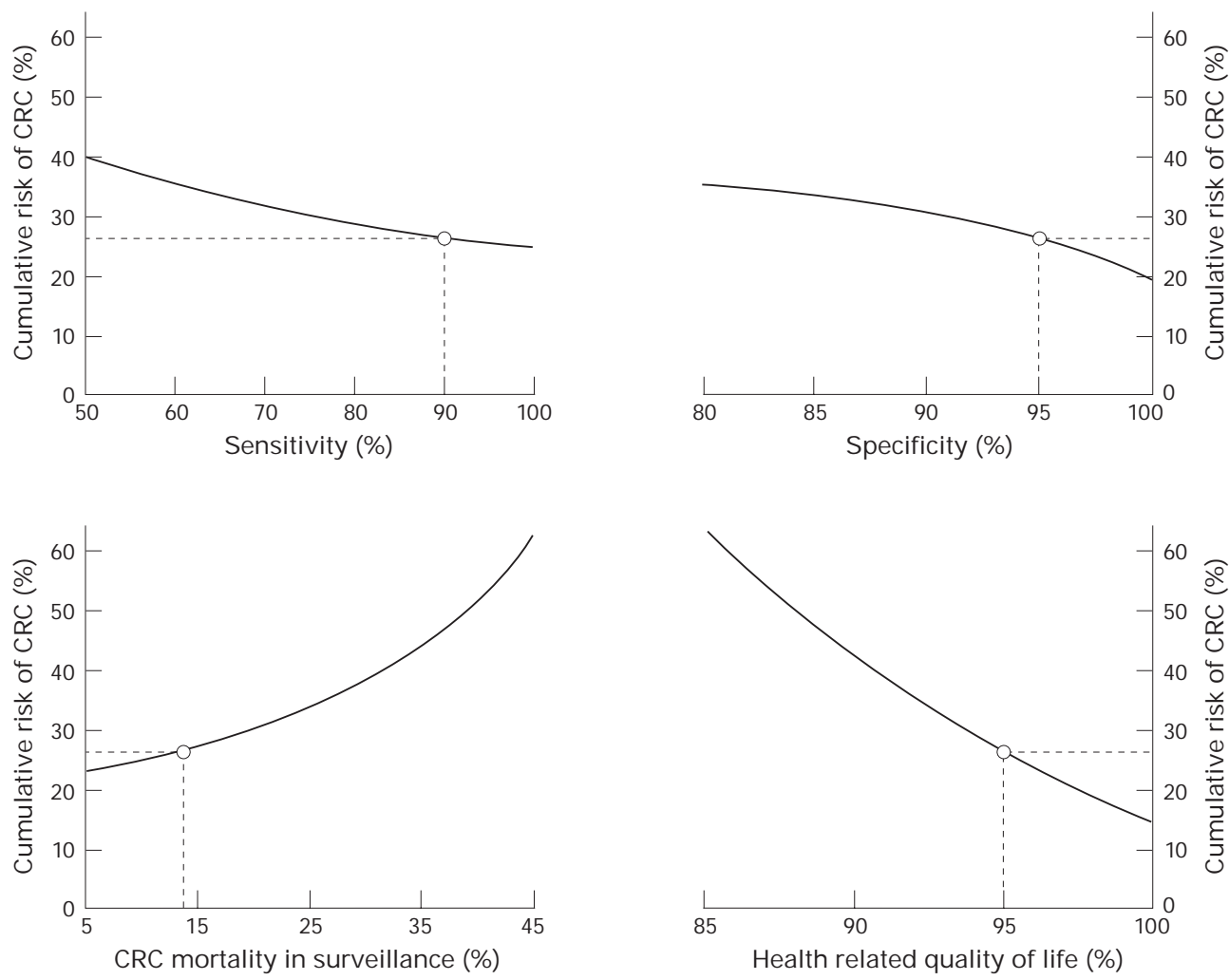

Figure 3 One way sensitivity analyses of the threshold value for cumulative cancer risk. CRC, colorectal cancer.

strategy based on biannual surveillance. As the threshold value is only slightly less than the actual cumulative cancer rate of $30 \%$, one may expect that slight variations of the assumptions built into the model will easily raise the threshold above the cumulative cancer rate.

The influences of the assumptions built into the model on the threshold value are tested in multiple sensitivity analyses, where each assumption is varied over a broad range. Compared with other parameters, the outcome of the model is less sensitive to the cost estimates used in the model. Smaller expenses for the endoscopic procedure change surveillance into a more favourable management strategy and reduce the threshold in its favour. For instance, varying the cost of colonoscopy between $\$ 200, \$ 500$, and $\$ 1500$ results in changes of the threshold value between $18 \%$, $21 \%$, and $35 \%$, respectively. A rise in the costs of proctocolectomy makes surveillance a less favourable strategy, while expensive terminal care has an opposite effect. For instance, halving or doubling the costs of proctocolectomy changes the threshold to $24 \%$ or $32 \%$, respectively. Halving or doubling the costs of terminal care changes the threshold to $29 \%$ or $23 \%$, respectively. As higher incomes translate into larger benefits associated with gained life years, an annual income of $\$ 50000$ leads to a threshold value of $19 \%$.

Any increase in the time lag between the first occurrence of dysplasia and cancer reduces the amount of life years saved. A long time lag results in a larger fraction of patients who undergo proctocolectomy only for dysplasia, but who would have remained cancer free for a long time period. Overall the influence of this phenomenon is relatively small, however. Varying the time lag from one to five years changes the threshold by only $1 \%$. If one assumes that the benefit of surveillance and its associated reduction in cancer related mortality remain unaffected by increasing the interval of surveillance, any decrease in the frequency of endoscopic procedures has an effect similar to reducing the costs of the endoscopic procedure. For instance, decreasing the frequency from once per two years to once per three or four years, decreases the threshold to $19 \%$ and $14 \%$, respectively, as it makes surveillance a more cost effective strategy.

The upper two panels of fig 3 display how the threshold is influenced by the sensitivity and specificity of colonoscopy plus biopsy in detecting dysplasia or cancer. Changes in the sensitivity by $10 \%$ have little influence on the threshold. As the sensitivity decreases, the fraction of false negative tests increases and more patients die from colorectal cancer. Changes of specificity by $5 \%$ also exert relatively little influence on the threshold. A decrease in the specificity leads to a larger fraction of false positive tests and more patients undergoing unnecessary proctocolectomy associated with a reduction in HRQL. Any type of less efficacious surveillance characterised by a low sensitivity or specificity value is associated with a higher threshold.

The lower two panels of fig 3 display how cancer related mortality associated with a surveillance programme and how reduction in HRQL after proctocolectomy affect the threshold. High mortality rates from colorectal cancer despite surveillance negate the benefit of any such strategy and raise the threshold. 


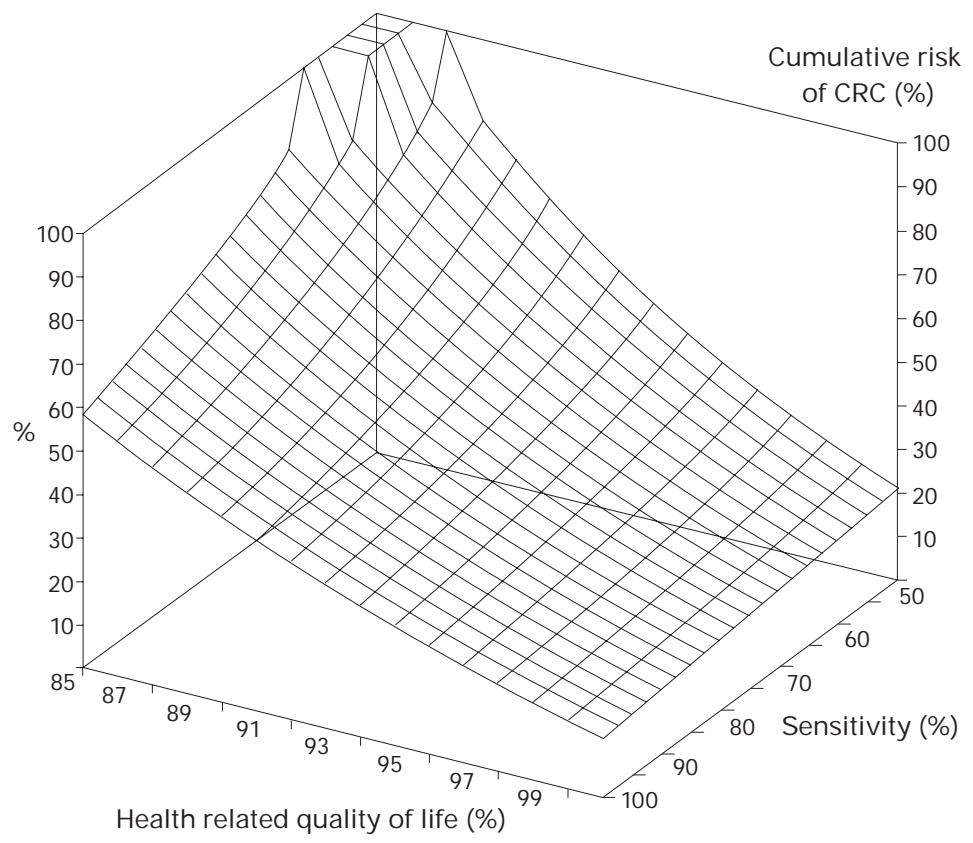

Figure 4 Two way sensitivity analysis of the threshold value for cumulative cancer risk. CRC, colorectal cancer.
HRQL $=98 \%$, sensitivity $=95 \%$, and specificity $=98 \%$. The resulting threshold value $\%$ CRC $=16 \%$ lies well below the cumulative lifetime probability. On the other hand, a set comprising of mortality $=16 \%$, HRQL $=$ $90 \%$, sensitivity $=85 \%$, and specificity $=90 \%$ is associated with a threshold of $59 \%$ outside the cumulative lifetime risk of most ulcerative colitis patients to ever develop colorectal cancer. Both sets of assumptions are by no means extreme and seem to fall within a reasonable range that might be expected by a widely distributed surveillance programme.

\section{Discussion}

The present cost-benefit analysis compares the outcomes of ulcerative colitis managed with or without endoscopic surveillance to prevent death from colorectal cancer. The analysis yields a threshold for the cumulative cancer risk, which would render surveillance colonoscopy the preferred management strategy. A low threshold speaks in favour of surveillance, whereas a high threshold argues against it. The calculated value for the threshold is compared with the actual risk of developing colorectal cancer. Beginning only 10 years after the onset of ulcerative colitis, the cumulative risk of colorectal cancer has been reported to reach $30 \%$ over the next 30 years, that is, 40 years after the first diagnosis of the disease..$^{4-7}$ Under baseline assumptions, the cost-benefit analysis yields a threshold of $27 \%$. Varying individual probability values and cost estimates can raise the threshold far above or lower it far below the true cumulative cancer risk. If several of the assumptions built into the model are varied jointly, even small changes can lead to extreme threshold values. As such sensitivity analyses show, one can conceive similarly reasonable sets of assumptions that result either in excessively high or low thresholds. The decision analysis fails to yield a clear cut answer about the usefulness of surveillance colonoscopy in ulcerative colitis.

Three prior decision analyses have also addressed the question of endoscopic surveillance in ulcerative colitis. Using a decision tree and threshold analysis, Gage compared the expected utilities of prophylactic proctocolectomy versus surveillance colonoscopy in a patient with ulcerative colitis since 20 years. ${ }^{27}$ The threshold indicated the cumulative risk of colorectal cancer, above which prophylactic proctocolectomy became the favoured management option. It varied between $16 \%$ and $89 \%$ depending on the postoperative mortality, as well as the sensitivity and specificity of the surveillance colonoscopy. Provenzale et al used a Markov model to assess the increase in life expectancy obtained through prophylactic colectomy, surveillance colonoscopy, and expectant management. ${ }^{15}$ In a cohort of 30 year old patients with ulcerative colitis since 10 years, prophylactic proctocolectomy increased life expectancy by $2-10$ months compared with surveillance, and by 1.1-1.4 years compared with expectant management. In a separate analysis, Provenzale et al calculated an incremental cost effectiveness ratio of $\$ 4700$ per life sively high or low thresholds. Figure 1 and show the baseline parameters. Simultan variations of all four parameters from fig 3 lead to a second set of values that is slightly different from the baseline set, such as mortality $=10 \%$, 
year saved through surveillance every five years, compared with a strategy of no surveillance. ${ }^{21} \mathrm{~A}$ decrease in the surveillance interval to four and three years resulted in incremental cost effectiveness ratios of $\$ 83400$ and $\$ 111600$, respectively, per additional life year gained. The authors recommended the implementation of a surveillance programme, because it would increase life expectancy and because its incremental cost effectiveness ratio compared favourably with screening strategies for tuberculosis or cervical cancer and other medical interventions, such as heart transplantation.

Our analysis differs from these previous studies in several aspects. Using the human capital approach, the cost-benefit analysis balances the cost of surveillance, surgery, and terminal care against the benefit of life years saved. ${ }^{24}$ Similar to the studies by Provenzale et al but different form the study by Gage, a Markov process is used to assess the time dependent behaviour of cancer risk in ulcerative colitis. ${ }^{1521}$ Rather than expressing our results as utilities, costs, or incremental cost effectiveness ratios, the outcomes of the competing treatment strategies are compared in terms of an overall threshold probability. The threshold value relates to a clinically available parameter, such as cumulative cancer risk in long standing ulcerative colitis. This relation between the threshold and the cancer risk makes it easier to appreciate the outcome of the analysis. The decision in favour or against surveillance does not need to rely on abstract economic cost parameters or extraneous comparisons with unrelated types of medical interventions.

Would another or more complex type of decision analysis yield a different outcome? A more detailed analysis could include, for instance, operative mortality associated with proctocolectomy, patient compliance with a surveillance programme, and the impact of surveillance itself on HRQL in ulcerative colitis. Rather than analyse the transition from normal colon to dysplasia, one could break down the single state of dysplasia into two separate states of low grade and high grade dysplasia. Similarly, the state of colorectal cancer could be broken down into cancers of different Dukes's stages. Modelling the natural history of colorectal cancer in a more detailed fashion is largely compromised by a lack of reliable clinical data for most of these additional states and their associated transition probabilities. Instead of adding precision to the model, any expansion of the set of assumptions built into the model would only increase its range of variation. The current set of parameters in the model can already lead to fluctuations of the threshold between $0 \%$ and $100 \%$.

Gastroenterologists by the nature of their profession like to do surveillance colonoscopy and tend to favour reports that recommend such strategy. Authors and readers alike prefer clear cut and easy decision rules. The term "medical decision analysis" alludes to a technique that can calculate the most appropriate decision rule. Unfortunately, medical decision analysis fails to resolve the clinical dilemma of endoscopic surveillance in ulcerative colitis. Small variations in several of the assumptions built into the model result in thresholds that seemingly speak in favour of or against surveillance. In order to provide more narrow estimates regarding the usefulness of surveillance, decision analysis would have to rely on very precise clinical evidence that is currently missing. None of several clinical trials has been able to provide unequivocal evidence in favour of surveillance. ${ }^{28-32}$ The major reason for this failure relates to the prohibitively large number of patients with ulcerative colitis who need to be followed over a prolonged time period, before statistically sound results could be obtained.

In conclusion, medical decision analysis fails to show that surveillance colonoscopy would be beneficial in long standing ulcerative colitis. Presently, there are also no clinical data to support the contention that surveillance colonoscopy is a cost effective means to prevent death from colorectal cancer in patients with ulcerative colitis. These statements are not meant to belittle the general contribution of colonoscopy in protecting against death from colorectal cancer. Patients do benefit from screening colonoscopy and colonoscopic pursuits of newly developed symptoms or suspicious findings. However, it is not possible to prove that frequent colonoscopies scheduled at regular intervals are an effective means to manage the increased risk of colorectal cancer associated with ulcerative colitis.

F Delco was supported by the Swiss Foundation for Grants in Medicine and Biology.

1 Greenstein AJ, Sachar DB, Smith H, et al. Cancer in univeral and left-sided colitis: factors determining risk. Gastroenterology 1979;77:290-4.

2 Devroede GJ, Taylor WF, Sauer WG, et al Cancer risk and life expectancy of children with ulcerative colitis. $N$ Engl 7 Med 1971;285:17-21.

3 Devroede G, Taylor WF. On calculating cancer risk and survival of ulcerative colitis patients with the life table method. Gastroenterology 1976;71:505-9.

4 Gyde SN, Prior P, Allan RN, et al. Colorectal cancer in ulcerative colitis: a cohort study of primary referrals from three centres. Gut 1988;29:206-17.

5 Gilat T, Fireman Z, Grossman A, et al. Colorectal cancer in patients with ulcerative colitis. A population study in central Israel. Gastroenterology 1988;94:870-7.

6 Maratka Z, Nedbal J, Kociánová J, et al. Incidence of colorectal cancer in proctocolitis: a retrospective study of 959 cases over 40 years. Gut 1985;26:43-9.

7 Lennard-Jones JE, Melville DM, Morson BC, et al. Precancer and cancer in extensive ulcerative colitis: findings among 401 patients over 22 years. Gut 1990;31:800-6.

8 Ries LAG, Kosary CL, Hankey BF, et al. SEER cancer statistics review, 1973-1994, NIH Publication No. 97-2789. Bethesda, MD: National Cancer Institute, 1997.

Choi PK, Kim WH. Colon cancer surveillance. Gastroenterol Clin North Am 1995;24:671-87.

10 Bauer WM, Lashner BA. Inflammatory bowel disease and intestinal cancer. Practical Gastroenterology November 1998:20-9.

11 Rex DK. Surveillance colonoscopy in ulcerative colitis. Clin Perspect Gastroenterol 1999;2:9-15.

12 Pauker SG, Kassirer JP. The threshold approach to clinical decision making. N Engl f Med 1980;302:1109-17.

13 Sonnenberg FA, Beck JR. Markov models in medical decision making: a practical guide. Med Decis Making 1993;13:332-8.

14 National Center for Health Statistics. US decennial life tables for 1989-91, Publication No. PHS-98-1150-1, Vol 1, No 1. Hyattsville, MD: DHHS, 1997.

15 Provenzale D, Kowdley KV, Arora S, et al. Prophylactic colectomy or surveillance for chronic ulcerative colitis? A decision analysis. Gastroenterology 1995;108:1188-96.

16 Ransohoff DF, Riddell RH, Levin B. Ulcerative colitis and colonic cancer. Problems in assessing the diagnostic colonic cancer. Problems in assessing the diagnostic
usefulness of mucosal dysplasia. Dis Colon Rectum 1985;28: user-8. 
17 Connell WR, Talbot IC, Harpaz N, et al. Clinicopathological characteristics of colorectal carcinoma complicating ulcerative colitis. Gut 1994;35:1419-23.

18 Lashner BA. Recommendations for colorectal cancer screening in ulcerative colitis: a review of research from a single university-based surveillance program. Am f Gastroenerol 1992;87:168-75.

19 Lashner BA, Hanauer SB, Silverstein MD. Optimal timing of colonoscopy in ulcerative colitis. Ann Intern Med 1988;108:274-8.

20 Haert R, Classen M. Complications of diagnostic gastrointestinal endoscopy. Endoscopy 1990;22:229-33.

21 Provenzale D, Wong JB, Onken JE, et al. Performing a costeffectiveness analysis: surveillance of patients with ulcerative colitis. Am $\mathcal{F}$ Gastroenterol 1997;93:872-80.

22 Köhler LW, Pemberton JH, Zinsmeister AR, et al. Quality of life after proctocolectomy. A comparison of Brook ileostomy, Kock pouch, and ileal pouch-anal anastomosis. Gastroenterology 1991;101:679-84.

23 Provenzale D, Shaerin M, Phillips-Bute BG, et al. Health-related quality of life after ileoanal pull-though: evaluation and assessment of new health status measures. Gastroenterology 1997;113:7-14.

F. Economic valuation of life and health In: Zweifel P, Breyer F, eds. Health economics. New York: Oxford University Press, 1997:27-8.
25 US Bureau of the Census. Statistical abstract of the United States: 1997, 117th ed Washington, DC: US Government Printing Office, 1997.

26 Weinstein MC, Stason WB. Foundations of costeffectiveness for health and medical practices. $N$ Engl f Med 1977;296:716-21.

27 Gage TP. Managing the cancer risk in chronic ulcerative colitis. A decision-analytic approach. I Clin Gastroenterol 1986;8:50-7.

28 Lashner BA, Kane SV, Hanauer SB. Colon cancer surveillance in chronic ulcerative colitis: historical cohort study. Am $\mathcal{F}$ Gastroenterol 1990;85:1083-7.

29 Choi PM, Nugent FW, Schoez DJ Jr, et al. Colonoscopic surveillance reduces mortality from colorectal cancer in ulcerative colitis. Gastroenterology 1993;105:418-24.

30 Lynch DAF, Lobo AJ, Sobala GM, et al. Failure of colonoscopic surveillance in ulcerative colitis. Gut 1993;34:107580 .

31 Connell WR, Lennard-Jones JE, Williams CB. Factors affecting the outcome of endoscopic surveillance for cancer in ulcerative colitis. Gastroenterology 1994;107:934-4.

32 Karlén P, Kornfeld D, Broström O, et al. Is colonoscopic surveillance reducing colorectal cancer mortality in ulcerative colitis? A population based case control study. Gut 1998;42:711-14. 